\title{
An Iterative Algorithm to Produce a Positive Definite Correlation Matrix from an "Approximate Correlation Matrix" (With a Program User's Guide)
}

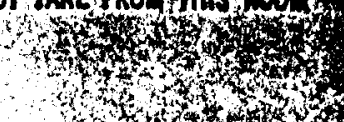

Ronald L. Iman, James M. Davenport

Prepared by

Sandla National Laboratories

Albuquerque, New Mexico 87185 and Livermore, Callfornia 94550

for the United States Department of Engroy

under Contract DE-AC04-76DPOO789

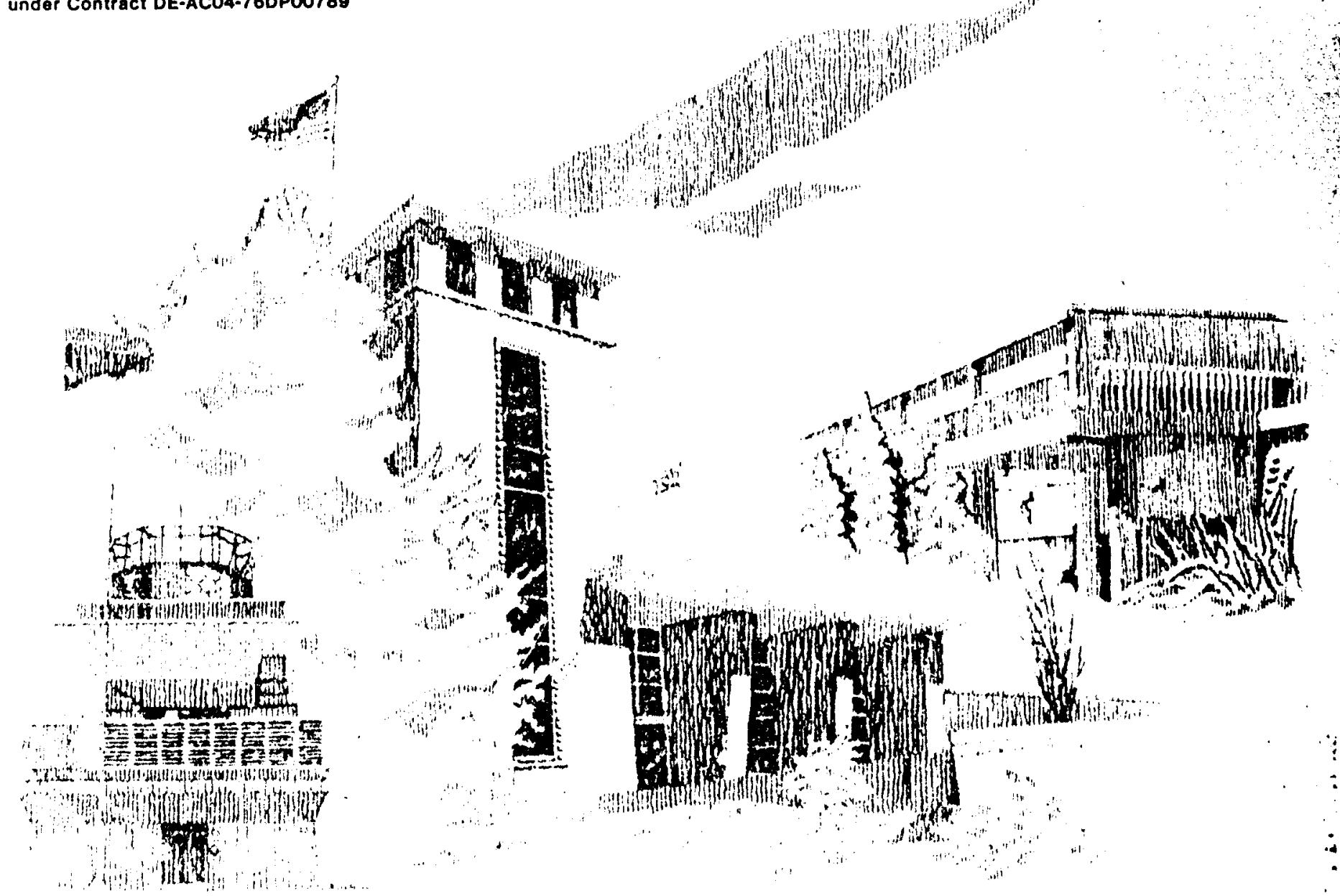


Ineved by Sandla National Laboratoriea, operated for the Unitod Statee Department of Bnersy by Bandin Corporation.

NOTICE: This report was propared as an nocount of wotk eponeored by an agency of the United States Covernment. Noither the Unitod Btates Govern. ment nor any areney theroof, nor any of thoir employeoen nor any of their contractors, subcontractora, or their employeen, makee euy werranty, exprese contractors, subcontractorn, or thoif amployes, mansibility for the eccuracy, or impliod, or asoumes any logal linbility or recponsibility for the accuracy. completenos, or usefulneas of any information, apparatus, product, or process disclosed, or represents that its uee would not infringe privately owned iscrice by trade name trademert, manufecturer, or otherwise, does not corve by trade necesthe by trad iny of their contractorn or aubeontractors. The viom and oplnions expreaced herein do not necesenrily etate or reflect thowe of the United Btates Government, any agency ther-of or any of their contractors or uubcontractora.

Printed in the United States of America Available from

National Technical Information Service

U.S. Department of Commerce

5285 Port Royal Road

Springfield, VA 22161

NTIS price codea

Printod copy: $\mathrm{A03}$

Microficho copy: A0I 
SAND81-1376

Unlimited Release

Printed June 1982

An Iterative Algorithm to Produce a Positive Definite Correlation Matrix from an "Approximate Correlation Matrix"

(With a Program User's Guide)

James $M$. Davenport

Department of Mathematics

Texas Tech University

Lubbock, Texas 79409

Ronald L. Iman

Statistics and Computing Division 1223

Sandia National Laboratories

Albuquerque, New Mexico 87185

\section{ABSTRACT}

This report contains an explanation of an algorithm that, when executed, will operate on any symmetric "approximate correlation matrix" by iteratively adjusting the eigenvalues of this matrix. The ohjective of this algorithm is to produce a valid, positive definite, correlation matrix. Also a description of a program (called POSDEF) which implements the algorithm is given. 
THIS PAGE LEFT BLANK INTENTIONALLY 


\section{Table of Contents}

Page

I. Introduction

II. Description of the Algorithm 6

III. Important Variations of the Algorithm 11

IV. Program Description 19

A. Limitations on the Program 19

B. Input-Parameter Cards 20

C. Explanation of Output 23

D. An Example with Selected Portions of the Output 24

V. Deck Setup for POSDEF 34

VI. Discussion $\quad 37$

Bibliography 41

Appendix A - Listing of the For'ran Source Deck for POSDEF 42 
THIS PAGE LEFT BLANK INTENTIONALLY 


\section{Introduction}

Many modeling situations exist where the assumption of independence among variables may not be appropriate. Indeed, "real world problems" exist where dependences or correlations do exist among the variables, and the design of an experiment investigating these variables should make use of this "correlation structure."

This dependence structure among the variables is appropriately summarized in a rank correlation matrix. Iman and Davenport (1980) discuss in some detail the advantages of using correlations, specifically rank correlations, as a method of inducing dependences among variables. Therefore, within this report, we assume that the dependence structure among the variables is adequately summarized in a rank correlation matrix.

In general, when we speak of correlations in this report, we will mean rank correlations, since it is not always appropriate to "talk about" a raw correlation. However, it should be pointed out that, if it is appropriate to define a Pearsonian correlation coefficient between two variables, then the methods discussed here apply without loss of generality.

It is extremely important that existing correlation structure be specified as accurately as possible. If the modeler is fortunate, he can estimate this correlation matrix from data, and hence, the resulting estimated correlation matrix is either positive definite or positive 
semi-definite. However, the modeler may find that no data are available, and he is faced with the task of assigning values to these correlation coefficients by whatever methods he can assemble. Under such conditions, it is not unusual for the modeler to use a "sophisticated guess" for the value of a correlation coefficient. Furthermore, the modeler seldom can "estimate" the entire correlation structure simultaneously and is compelled to provide a collection of pair-wise correlation coefficients. That is, the modeler specifies this correlation matrix by setting the diagonal el ements equal to +1.0 and each off diagonal el ement equal to some number between -1.0 and +1.0 , that he feels best describes the dependence between those two variables.

Such a collection of pair-wise correlation coefficients does not a "correlation matrix" make! Indeed, Mother Nature does not always have a charitable character, and it is not too surprising that such "correlation matrices" are not positive definite. Therefore, the modeler must deal with the problem that he cannot assign values indiscriminately to the off-diagonal elements without regard for the total correlation structure.

Such an example is the case of the multivariate $k$-dimensional normal distribution. Let $\Sigma_{\rho}$ be the correlation matrix of this distribution such that all off-diagonal elements are equal to $\rho$ (the diagonal elements are, of course, equal to +1.0$)$. Then it can be shown that

$$
-\frac{1}{k-1}<\rho<1
$$


That is, as $k$ becomes large, then there can be no large negative correlations.

The intent of this report is to present an algorithm (and a program) that will take the collection of pair-wise rank correlations (in the sequel, called the "approximate correlation matrix") and iteratively adjust this matrix until it is positive definite (note that the approximate correlation matrix is symmetric).

The algorithm given in this report is based on the premise that an approximate correlation matrix that is constructed by the method described above will have the following structure. Some of the eigenvalues will be positive. In fact, if the modeler has been careful in the selection of the pairwise correlations, taking into consideration the total correlation structure, then all of these eigenvalues will be positive; and hence the correlation matrix is positive definite. However, it may be possible for some of the eigenvalues to be zero or negative. If there are negative eigenvalues present and if one or more of those negative eigenvalues are relatively large in absolute value (say a value 1,2 or 3 ), then we say that this matrix is illconditioned with respect to this algorithm.

We have investigated several examples of this type of approximate correlation matrix, and have noted the following additional patterns. The number of negative eigenvalues was relatively small as compared to the dimension of the matrix. Also the values of these negative eigenvalues were very near zero. In addition, some of the positive eigenvalues were very near zero.

We feel that this structure that we have observed in these examples 
is related to the multicollinearity problem that is common in regression analysis. That is, as the dimension of the matrix (the number of variables) increases, then the associated correlation matrix is more likely to be near singular. The present problem is made more complicated by the fact that some negative eigenvalues are also present.

If the eigenvalues that are negative are few in number and small in absolute value, then it should be possible to adjust them so that the initial matrix is transformed into a positive definite matrix (all eigenvalues positive), subject to the coristraint that the values of the correlation coefficients in the new matrix are very close to the values in the initial approximate correlation matrix. While we present no theorems in this report indicating the conditions necessary and/or sufficient under which this algorithm will be valid, our experience in working with this type of matrix indicates that it will converge (i.e., we have not encountered any examples where it failed to converge.)

Likewise, our investigations of the several examples that we considered indicate that this iterative adjustment scheme produces small changes in the values of the correlation coefficients in the initial approximate correlation mairix. This is due to the fact that, for this type of matrix, small changes in the eigenvalues produce small changes in the entries of the matrix.

In Section II, we describe the algorithm that is used in the program POSDEF in detail. However, there are some very important variations to this algorithm that merit our attention. These are discussed in section 
$-5-$

III. A description of the program POSDEF is given in section IV, and an example of the deck setup for using POSDEF is in section $V$. The final section contains a discussion. 


\section{Description of the Algorithm}

Let $C$ be an $n \times n$ symmatric, non-singular matrix such that the elements of C satisfy the following:

$$
\begin{aligned}
& \quad c_{i j}=1 \quad i=1,2, \ldots . n \\
& \text { and }-1<c_{i j}<1 \text { for } i \neq j .
\end{aligned}
$$

Then it is well known that there exists a matrix $Z$ and a diagonal matrix $D$, each of dimension $n \times n$ such that (see Graybill, 1961)

$$
\underset{\sim}{C Z}=\underset{\sim}{Z} \text {. }
$$

The columns of $Z$ are the respective eigenvectors of the matrix ${ }_{\sim}$ and the elements of $D$ are the eigenvalues. That is,

$$
\tilde{D}=\left[\begin{array}{llllll}
\lambda_{1} & & & & & \\
& \lambda_{2} & & & \\
& & \lambda_{3} & & \\
& & & \cdot & \\
& & & & \cdot & \\
& & & & \lambda_{n}
\end{array}\right]
$$

and we assume, without loss of generality, that $\lambda_{1}<\lambda_{2}<\ldots<\lambda_{n}$. Since $Z$ is an orthogonal matrix, we have the following result. 


$$
\begin{aligned}
& \underset{\sim \sim \sim}{\mathrm{CZZ}^{\circ}}=\underset{\sim \sim \sim}{\mathrm{ZDZ^{- }}} \\
& \underline{C}=\underset{\sim}{\mathrm{ZD} Z^{\prime}} \\
& \underline{c}=\sum_{i=1}^{n} \lambda_{i} Z_{i} Z_{i}
\end{aligned}
$$

Where $z_{i}$ is the $i^{\text {th }}$ column of $z$.

Now suppose that $\lambda_{1}<\lambda_{2}<\ldots<\lambda_{k}, k<n$ are all negative, hut smail in absolute value. The first step in the algorithm is to change each of these negative eigenvalues to a small positive number that we will denote by $\varepsilon$. The idea is to make all of the eigenvalues positive, and hence, the new matrix to be calculated should be positive definite, or at least have fewer negative eigenvalues than it had previously. However, we do not wish to change the values of the individual elements of $C$ anymore than we have to. Therefore we choose a value for $\varepsilon$ that is very sinall, namely $\varepsilon=0.001$. Likewise, we choose not to change the eigenvectors, to minimize the effect on the matrix $\mathbf{c}$.

The second step in the algorithm is to examine the eigenvalues $\lambda_{k+1}<\lambda_{k+2}<\ldots<\lambda_{2 k}$ and change these values, if necessary, by the following procedure.

$$
\lambda_{i}{ }^{*}=\left\{\begin{array}{l}
\varepsilon \text { if } \lambda_{i}<\varepsilon \\
\lambda_{i} \text { if } \lambda_{i}>\varepsilon
\end{array} \quad \text { for } i=k+1, \ldots, 2 k .\right.
$$


That is, if $\lambda_{j}$ where $k+1<i<2 k$ is smaller than $\varepsilon=0.001$, then we increase its value to .001 . If it is larger than $\varepsilon$, then we leave $\lambda_{j}$ unchanged. This portion of the algorithm improves the operating characteristics of the overall iteration scheme in the examples that we considered. This is discussed in more detail in Section III.

Now a new matrix can be calculated using the new eigenvalues discussed above and is defined as follows:

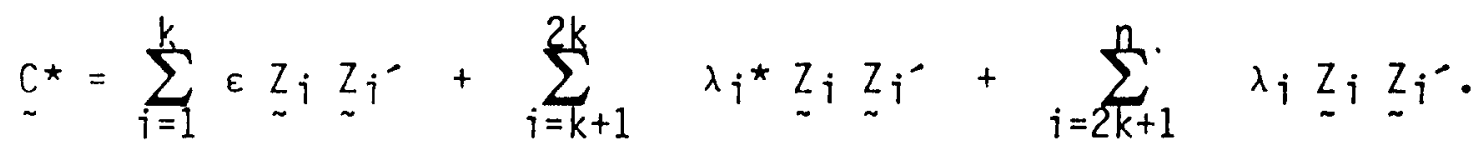

Recall that the original matrix $\mathrm{C}$ had diagonal elements equal to +1 . This will not be true of the matrix $C^{*}$. In general, the diagonal tlements of $C^{\star}$ will be slightly greater than one, and in fact, some of the off-diagonal elements of $\hat{c}^{\star}$ may be greater than one, depending upon the initial values within the matrix $C$.

Our objective is to produce a positive definite correlation matrix; therefore, some scaling of the elements of $c^{\star}$ must be done in order to make it conform to the definition of a correlation matrix. The procedure used here is to simply replace each of the diagonal elements of $c *$ by +1 and leave the off-diagonal elements unchanged. The result is a symmetric matrix with diagonal elements equal to positive one and off-diagonal elements approximately equal to those in $C$, the original matrix. We denote this new matrix by $C_{1}$ (indicating the end product of one cycle in the iteration process). 
If $\mathrm{C}_{\mathcal{1}}$ is positive definite then the process is complete and no further adjustment is needed. If the matrix $\mathrm{C}_{1}$ is not positive definite, then the entire process is repeated using the new matrix ${\underset{\sim}{1}}_{1}$ in place of the original matrix $C$. The end product of the second cycle of the iteration process is denoted by $\underset{\sim}{\mathrm{C}_{2}}$. If $\underset{\sim}{\mathrm{C}_{2}}$ is positive definite, then the process is complete. If $\mathrm{C}_{2}$ is not positive definite then the entire process is repeated with a third cycle. This iteration process is executed as many times as necessary, until the end product is a positive definite matrix.

A disclaimer is in order. We do not know whether or not this algorithm will always converge to a positive definite matrix. As stated in a previous secticn, we have no theorems that indicate when it will converge and/or when it will fail. However, we have used this algorithm on many approximate correlation matrices and it has never failed to converge to a positive definite matrix.

Given that the algorithm does converge to a positive definite matrix (i.e., ail of the eigenvalues are positive), then it would appear that there is a possibility of one or more of the off-diagonal elements having an absolute value larger than 1. The following theorem demonstrates that this is impossible.

Theorem. Given that the algorithm converges to a positive definite matrix A (of dimension $n \times n$ ) then $\left|\rho_{i j}\right|<1$ for all $i \neq j$ and $i, j=1,2, \ldots, n$ where $\rho_{i j}$ is the $(i j)^{\text {th }}$ element of $A$.

Proof: Assume $\rho_{i j}$ is an arbitrary but fixed element of $A$ such that $i \neq j$. Furthermore, assume $\left|\rho_{i j}\right|>1$. The matrix A can be transformed 
into a matrix $B$ by elementary row and column interchanges so that $b_{12}=b_{21}=\rho_{i j}=\rho_{j i}$. That is

$E_{j} E_{i} A E_{j^{\prime}} E_{j^{\prime}}=B=$

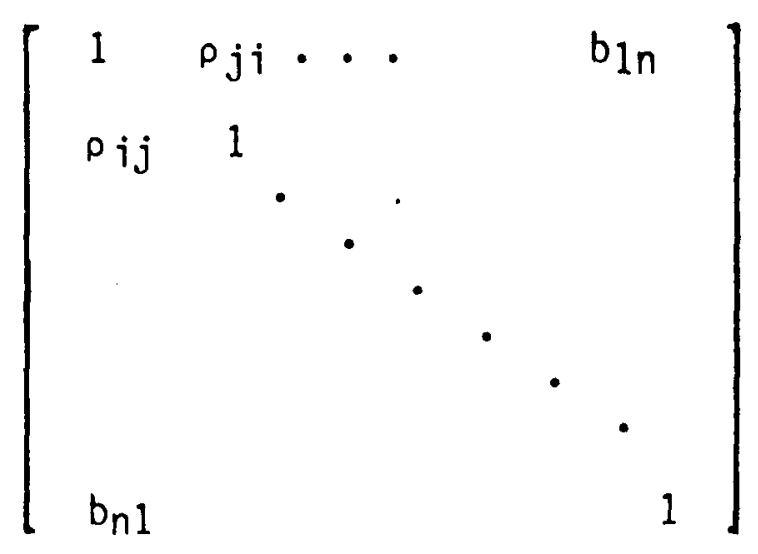

where $E_{j}$ and $E_{j}$ are the appropriately defined nonsingular matrices that accomplish the row and column interchanges. Note that the matrices $A$ and $B$ have identical eigenvalues (Graybill, 1961, Theorem 1.28, p.4). Further note that the matrix $B$ is not positive definite, since $\left(1-\rho_{i j}{ }^{2}\right)<0$ (Graybill, 1961, Theorem $1.23, p .3-4)$. Therefore, at least one of the eigenvalues of $B$ is zero or negative. But this contradicts the fact that all of the eigenvalues of the matrix $A$ are positive. Therefore, $\left|\rho_{i j}\right|<1$ for all $i \neq j$.

Hence, if the algorithm converges, then the matrix produced will indeed be a positive definite correlation matrix. The user should note that, in general, this matrix still will be very nearly singular. Likewise, our experience has shown that the changes in the values of the elements of the original matrix will be small (the reader is referred to Table 1 for some examples). In conclusion, the algorithm meets the objectives that we set at the outset. 
III. Important Variations of the Algorithm

There are essentially two important steps in this adjusting algorithm. They are:

(1) adjust the values of the eigenvalues $\lambda_{1}, \lambda_{2}, \ldots, \lambda_{2 k}$ associated with the matrix ${\underset{\sim}{i}}_{i}, i=0,1,2, \ldots$

(2) scale the diagonal and off-diagonal elements of the matrix $C_{i}{ }^{*}, i=0,1,2, \ldots$

See Section II for the respective definitions of ${\underset{\sim}{j}}_{j}$ and $C_{j}{ }^{*}$.

Several different schemes for accomplishing (1) were investigated before deciding to use the particular scheme described in section II. All of these were motivated by the following discussion.

Recall that we are assuming $\lambda_{1}, \lambda_{2}, \ldots, \lambda_{k}$ are all negative * and small in absolute value. Any such adjustment scheme must change these to positive values. However, we do wish to minimize the effect on the elements of the original matrix, ${\underset{\sim}{j}}_{i}$. Therefore, it seems reasonable to adjust $\lambda_{k+1}<\lambda_{k+2}<\ldots<\lambda_{2 k}$ accordingly to compensate for changing $\lambda_{1}<\lambda_{2}<\ldots<\lambda_{k}$. The objective is to minimize the net effect on the original matrix $\boldsymbol{C}_{i}$.

Since $\lambda_{1}$ is the smallest eigenvalue, we arbitrarily pair $\lambda_{1}$ with $\lambda_{2 k}$, the largest eigenvalue among the eigenvalues considered. Likewise pair $\lambda_{2}$ with $\lambda_{2 k-1}$ etc., and finally pair $\lambda_{k}$ with $\lambda_{k+1}$. Consider the pair $\left(\lambda_{1}, \lambda_{2 k}\right)$. Recal? that we will replace $\lambda_{1}$ 
with $\varepsilon$ and now wish to change $\lambda_{2 k}$ to a value, say $u$, such that

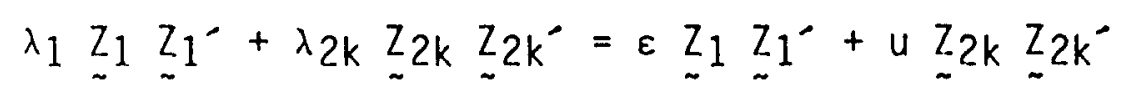

However, in general, this is impossible as no such value of $u$ exists that satisfies all of these equations. So to obtain some "overall effect," we can sum these equations to form one equation in one unknown. Prerultiplying the above equation by $\underset{\sim}{\mathcal{\prime}}$ and post-multiplying by $\underset{\sim}{J}$, where $\checkmark$ is a vector of all ones, yields

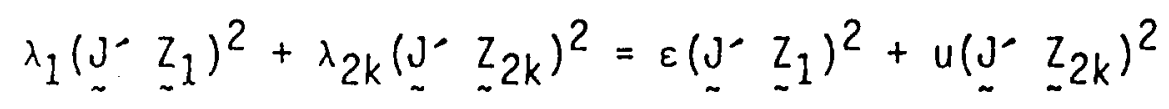

Solving for $u$, we have

$$
u=\left(\lambda_{1}-\varepsilon\right) R+\lambda_{2 k}
$$

where

$$
R=\left(J-z_{1}\right)^{2} /\left(j-z_{2 k}\right)^{2}
$$

Examination of equation 3.1 indicates that the essence of the adjustment to $\lambda_{2 k}$ is to decrease its value by some appropriate amount. Therefore, rather than using orily equation 3.2 for the definition of $R$, we investigated many different ways of finding a suitable value of $R$ including several obvious values such as zero and one. 
of course, it should be obvious that the total scheme of adjusting these eigenvalues would be accomplished on each pair of eigenvalues, $\left(\lambda_{1}, \lambda_{2 k}\right),\left(\lambda_{2}, \lambda_{2 k-1}\right), \ldots,\left(\lambda_{k}, \lambda_{k}+1\right)$.

Note that any choice of $R \neq 0$ may produce a negative value for $u$ (see equation 3.1), and hence this scheme would replace a positive eigenvalue with a regative rumber. This is obviousiy unacceptable, so we incorporated the following check into the algorithm.

$$
\operatorname{IF}(u \cdot L T \cdot \varepsilon) \quad u=\varepsilon
$$

Hence, if $R=0$ and statement 3.3 is included in the at orithm, the net effect is to replace those eigenvalues, among the set $\left(\lambda_{k+1}\right.$, $\left.\lambda_{k+2}, \ldots, \lambda_{2 k}\right)$, that are less than $\varepsilon$ with the value of $\varepsilon$. On the surface, this may seem strange to leave statement 3.3 in the program when $R=0$. Especially, since the eigenvalues $\lambda_{k+1}, \ldots, \lambda_{2 k}$ should be left unchanged, whenever $R=0$ (according to the equation 3.1). But empirical investigations indicated that leaving statement 3.3 in the algorithm whenever $R=0$ slightly improved the convergence characteristics of this iterative algorithm. That is, the algorithm converged in slightly fewer iterations whenever statement 3.3 remained in the a) gorithm.

At the same time we investigated the effects of statement 3.3 being included or excluded from the program, we were investigating many nonzero values of $R$. Also we investigated several techniques of "estimating" 
$R$ from the eigenvectors $z_{j}, i=1,2, \ldots, 2 k$. However, none of these produced any significant improvement in the algorithm over the choice $R=0$.

In conclusion, the algorithm as stated in Section II amounts to setting $R=0$ and including statement 3.3 in the program.

As stated at the beginning of this section, the second important step of this algorithm is to scale the elements of the matrix $C_{i}{ }^{\star}, i=0,1,2, \ldots$. . We tried five different methods of scaling this matrix and outline these in the following paragraphs.

\section{Method A}

The diagonal elements of the matrix are set equal to 1 . No other changes are made.

\section{Method B}

The diagonal elements of the matrix are set equal to 1 . Since some of the off-diagonal elements may be greater than or equal to one or less than or equal to minus one, it would seem that these elements should be scaled also. These off-diagonal elements are set equal to +.9999 and -.9999 respectively.

\section{Method C}

Each element in a row is divided by the square root of the diagonal element of that row. Likewise, earh element in a column is divided by the square root of the diagonal element of that column. That is, 


$$
r(i, j) \text { replaced by } \frac{r(i, j)}{\sqrt{r(i, j)} \cdot \sqrt{r(j, j)}}
$$

where $r(i, j)$ is the $(i, j)$ th element of the matrix under consideration. Note that this method guarantees that the diagonal elements will all be equal to 1 . However, this does not insure that the off-diagonal elements will be between minus one and plus one.

Method D

Essentially, this method is the same as method $C$ with the following exception. If a row (column) does not have any off-diagonal elements outside the interval $(-1,1)$, then the division process described in method $C$ is not accomplished. However, some of the diagonal elements may still be greater than 1 or less than 1 . Therefore, we follow the needed divisions described above, by setting all of the diagonal elements equal to 1.

Method E

This method is the same as method $D$ with the additional modification that whenever you do divide a row (column) by the square root the diagonal element of that row (column), you subtract 0.05 from the diagonal element hefore you take its square ront. For example, suppose $r(4,4)=1.0785$ and the division process is called for. Then every element in the fourth row and fourth column is divided by $\sqrt{1.0285}$. Then, as in method $D$, every diagonal element is set equal to 1. 
These different methods of scaling the matrix produced some interesting results. For example, method $C$ always converged in exactly one iteration on every exariple that we tested. However, method $C$ also produced the greatest change in the "correlations" in the original matrix $C_{i}$ (see Table 1). For this reason, we dacided not to use method $C$ as the scaling scheme.

Several examples of approximate correlation matrices were tested using the algorithm with all combinations of the eigenvalue adjustments, the five scaling schemes, and with or without statement 3.3. One such matrix is given as an example in Figure 1 . Within this matrix we selected two elements, namely $\rho_{12}=.9000$ an $\rho_{15,14}=.9999$ and have summarized the effect of the iteration procedure on these two elements in Table 1.

As stated earlier, we investigated many techniques for seleciing a value for $R$, but could find no value of $R$ that offered any significant improvement over the choice $R=0$. In Table 1 , we present the two values $R=0$ and $R=1$.

Further investigation of the examples, whenever $R=0$, indicated that the scaling scheme $A$ seems to produce the smallest changes in the values within the original matrix. This is illustrated with the two cases given in Table 1.

Finally our investigations indicate that, whenever $R=0$, the algorithm converged in fewer iterations with statement 3.3 included. Again, this is illustrated in Table 1. 
Our recommendation to use $R=0$, include statement 3.3 in the algorithm and use method $A$ for the scaling scheme. This is the algorithm described in section II. 
TABLE 1

COMPARISON OF DIFFERENT ADJUSTMENT AND SCALING SCHEMES

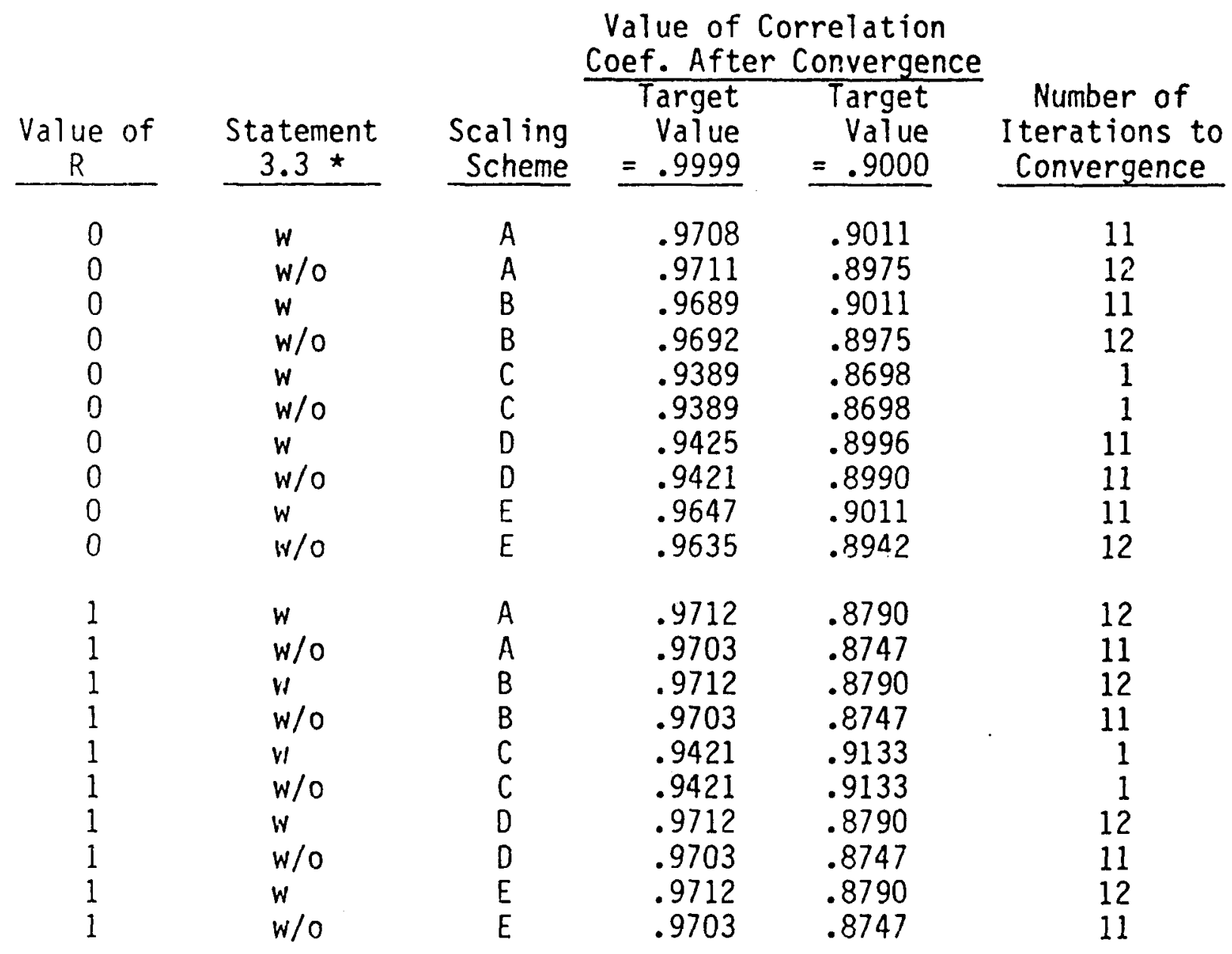

* - $w$ indicates that statement 3.3 is included, $w / 0$ indicates that it was excluded. 


\section{Program Description}

The description contained herein details how to use the program, called POSDEF, that implements the algorithm described in section II. This program has been developed at Sandia Laboratories and a complete listing of the Fortran computer code is given in Appendix A.

The procedure for the program is basically very simple. The "approximate correlation matrix" is read into the program. This matrix is adjusted iteratively until it converges to a positive definite matrix or until a fixed number of iterations have been e:secuted. In the following, we discussed the limitations on the program, input parameter card requirements, an explanation of the output, and an example with selected portions of the output.

\section{A. Limitations on the Program}

As the program presently exists, there are several limitations. However, these are not absolute limitations and can be changed by the user to suit his/her needs.

The first limitation concerns the upper limit on the size of the correlation matrix that can be read into the program. At present, a matrix of dimensions less than or equal to $20 \times 20$ can be accommodated. In fact, the program expects this matrix to he in symmetric storage notation. This is explained in the next subsection.

The second limitation concerns the value used for $\varepsilon$. Within the program, this is designated by the variable EIG. The value used for this 
quantity is 0.001 . This value can be changed without adversely affecting the program, but the user should realize that if the value of EIG is increased, this will increase the number of iterations required for convergence and cause greater perturbations in the individual elements of the original matrix.

The third limitation concerns the upper limit on the number of iterations allowed. The program presently allows a maximum of 20 iterations. If the algorithm does not converge after 20 iterations, the user should examine the original input "approximate correlation matrix" very carefully for unl ikely configurations of correlation structure. This is discussed in more detail in section VI.

The fourth limitation concerns the need for the IMSL subroutine EIGRS. The program POSDEF begins by decomposing the input matrix into its unique eigenvalues and eigenvectors. The IMSL subroutine will provide these eigenvalues and eigenvectors for an input matrix that is assumed to be in symmetric storage notation. If the EIGRS subroutine is not available, then some other subroutine that computes eigenvalues and eigenvectors should be used in its place.

B. Input - Parameter Cards.

All of the following parameter cards are required and must be in the order given. Also each card must conform to the format given. An explanation and illustration of each parameter card follows. 
1. MATRIX SIZE AND OUTPUT CONTROL CARD - Format (2I5).

This card has two integer valued arguments specified as shown below:

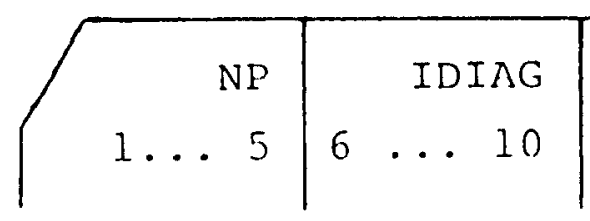

where

NP is the dimension of the "approximate correlation matrix" that is to be read in (NP - a maximum of 20).

IDIAG is an indicator variable that controls the printing of some of the output. After the last iteration the user may wish to see an analysis each $p \times p$ prime diagonal submatrix. See the next subsection for further discussion.

$=0$ then this analysis will not be done.

$=1$ then this analysis will be done and printed.

2. VARIABLE IDENTIFICATION CARD - Format (16I5)

This card reads in integer identification numbers that are associated with the variables that are represented by the "approximate correlation matrix." For example, suppose we wish to correlate the third, fourth and seventh variable out of ten variables being investigated. Then we would wish to label these variables as "3", "4", and "7" respectively. The following 
example illustrates this and note that a maximum of 20 labels may be read in.

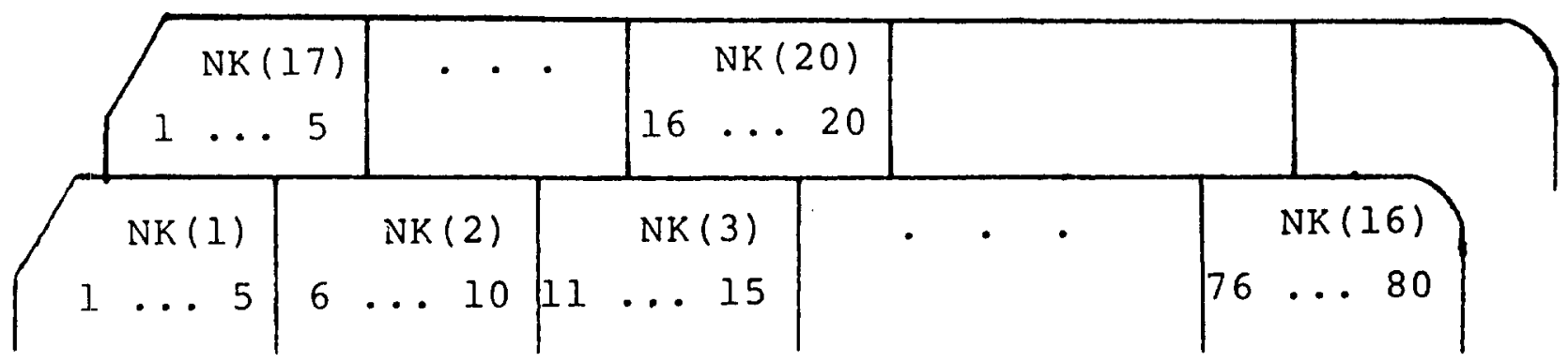

The only purpose of these identification numbers is to label the variables in the output. The algorithm does not depend on these numbers in any way.

3. "APPROXIMATE CORRELATION MATRIX" CARD - Format (8G10.4)

The program is formated such that this matrix must be in symmetric storage notation. That is, the symmetric matrix C is read in as a vector where the first element of the vector is $c(1,1)$, the second is $c(2,1)$, the third $c(2,2)$, the fourth $c(3,1)$, etc. As many cards as are necessary may be used as long as each card is in an 8G10.4 format. An example follows.

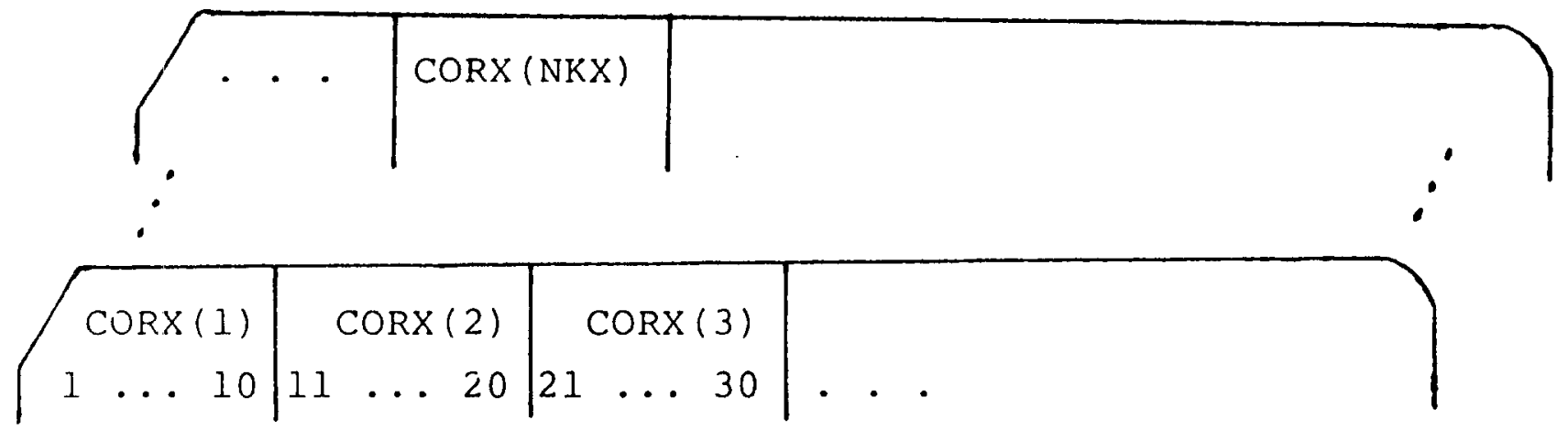

where NKX $=N P(N P+1) / 2$. This is the last input-parameter card. 
C. An Explanation of the Output.

A complete set of the following information is printed for each and every iteration.

1. The iteration number.

2. The input "approximate correlation matrix."

3. The eigenvalues and eigenvectors that are computed by the IMSL subroutine EIGRS. Note that the first row printed is actually the first column of $Z$ (see section II). That is, the first row printed is $z_{1}{ }^{\prime}$. In general, the $i^{\text {th }}$ row printed is $z_{i}{ }^{\prime}$, the $i$ th column of $z$.

4. The eigenvalues after the adjustment scheme has been executed (see sections II and III).

5. After the eigenvectors and the adjusted eigenvalues are multiplied appropriately to reconstruct the "new approximate correlation matrix," $\mathrm{C}^{\star}$, this matrix is printed.

6. This is followed by two statements indicating that the diagonal elements of $C^{*}$ are set equal to one. This completes a single iteration.

This information is printed for each iteration. If the algorithm converges on the $m^{\text {th }}$ iteration; then iteration $m+1$ will be begun, but the program will detect that all the eigenvalues are positive. Then the program will indicate that all the eigenvalues were positive on iteration $m+1$. This is followed by the final positive definite correlation matrix. 
If IDIAG $=0$ (see input - parameter card \#1) then the following information will not be printed. If IDIAG $=1$, then each $p \times p$ main diagonal submatrix $p=2, \ldots, N P$ is examined via its eigenvalues, eigenvectors and determinant. This completes the explanation of the output.

D. An Example with Selected Portions of the Output

In the following example, 16 of 64 different variables were to be correlated. The modeler provided a collection of pairwise rank correlations, that when taken as a "correlation matrix" was not positive definite. The labels for these 16 variables are $4,8,12, \ldots, 64$. The deck set-up for this example is given in section $V$ and the remainder of this example is best illustrated by the example output that follows.

Figure 1 gives the input "approximate correlation matrix." This is also the first page of the output of the program.

Figure 2 gives the eigenvalues, eigenvectors and determinant of this matrix. The eigenvalues after adjustment are also given. This is the second page of output.

Figure 3 gives the reconstructed "approximate correlation matrix" using the adjusted eigenvalues. Note that the diagonal elements and at least one of the off-diagonal elements is greater than one. This is also the third page of output.

Figure 4 gives the resulting "approximate correlation matrix" that is the end product of the first iteration. This matrix is also the 
beginning matrix of the second iteration. This is the fourth page of output from the program.

Figure 5 presents the input matrix to the $12^{\text {th }}$ iteration. Figure 6 presents the eigenvalues and eigenvectors of this matrix; as you can see, all the eigenvalues are positive and the determinant is positve. Therefore, the algorithm converged to this matrix after eleven iterations.

Figures 7 and 8 give respectively the eigenvalue/eigenvector analysis of the $2 \times 2$ and $3 \times 3$ prime diagonal submatrices of the matrix given in Figure 5. This is done for each prime diagonal submatrix as $p=2, \ldots, 16$.

This completes the explanation of this example and illustrated output. 
INPLT - RAMK CORRELATION STRUCTURE- FOR THE RANOOH VARIABLES BEING TRANSFORMED

\section{YAR LABLE NUMBER}

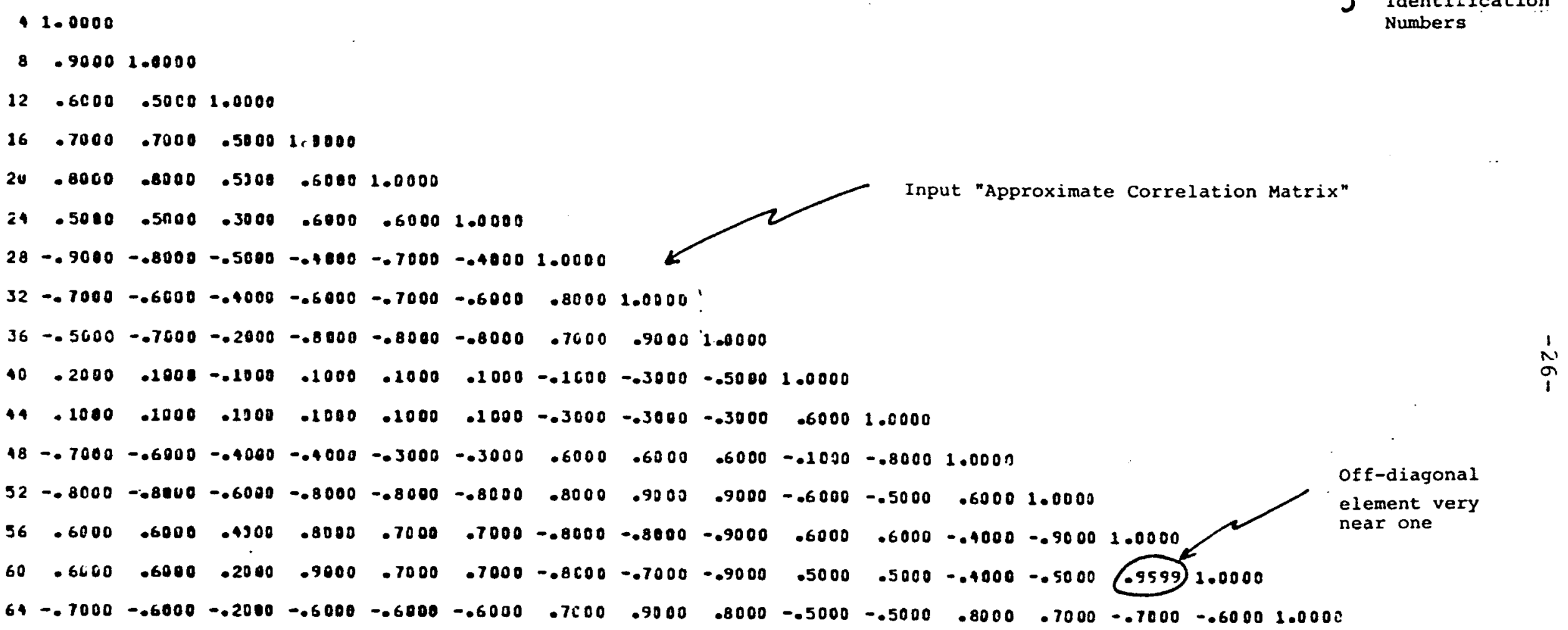


ANALYSIS CF IHE 16 BY IS PRINE OIAGONAL SUBMATRIX

PERF OAMANCE 1 NOEX $=.132\}$ Information from Subroutine EIGRS - See the IMSL Manual

EIGENVAL UES $=$

- Five negative eigenvalues

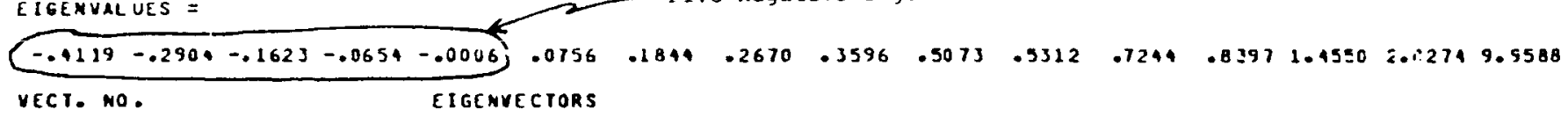

VECT. NO

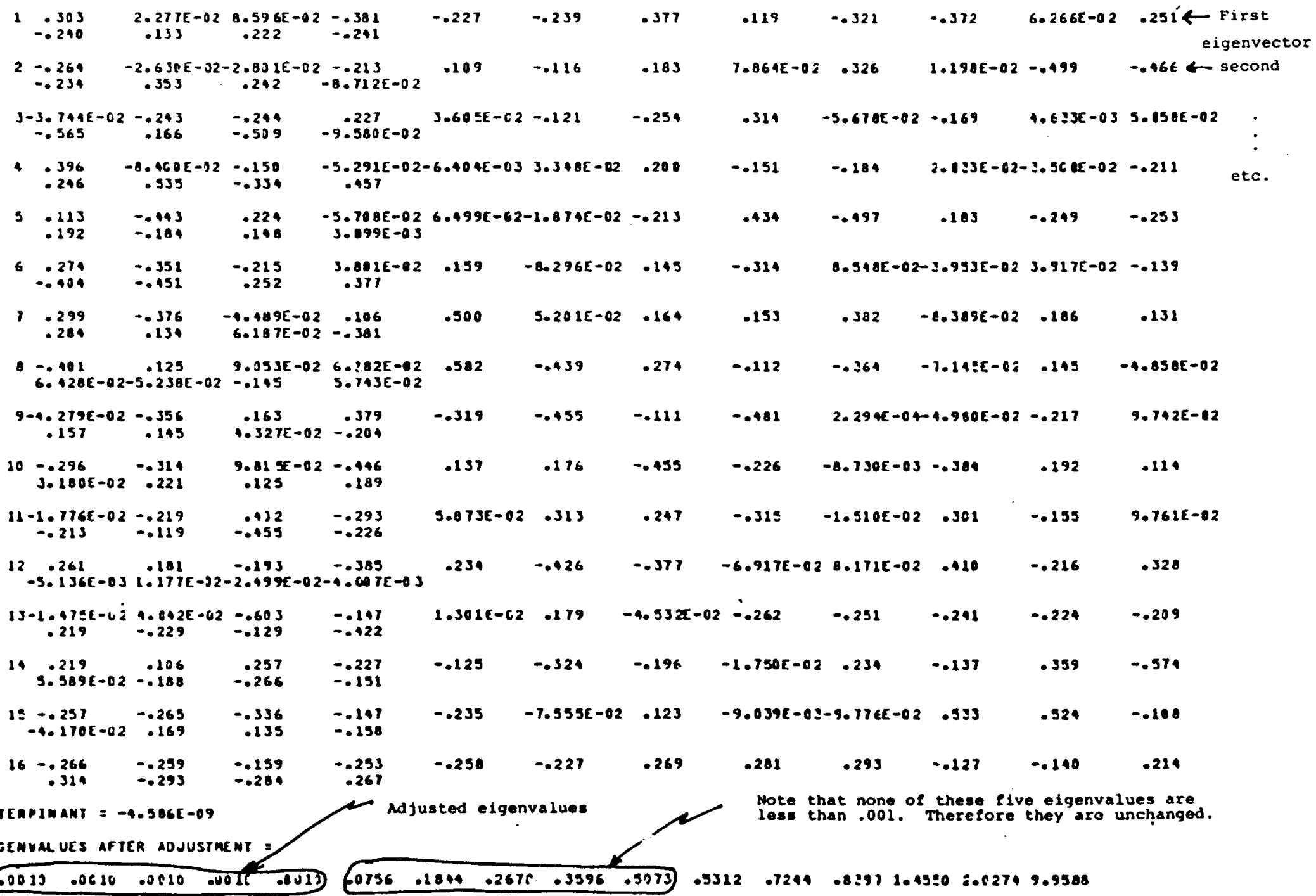




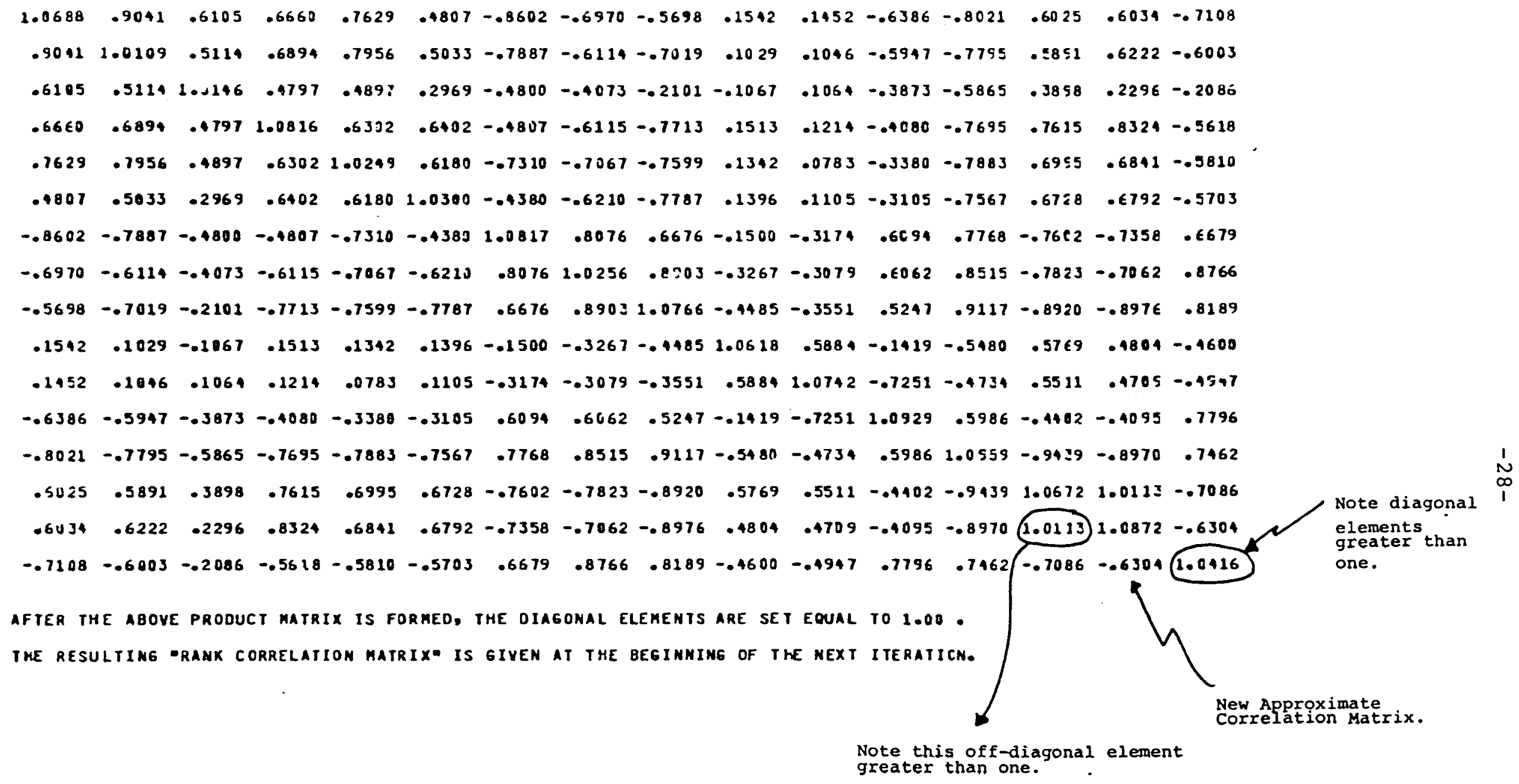
AFTER THE ABOVE PRODUCT MATRIX IS FORRED, THE DIAGONAL ELERENTS ARE SET EQUAL TO 1.00 THE RESULTING -RANK CORRELATION MATRIX- IS GIYEN AT THE BEgINMIMG OF THE NEXT ITERATICN. 
IAPIT - RAMK CORRELATION STRUCTURE FOR THE RANDCM VARIABLES BEIMG TRANSFOPMED

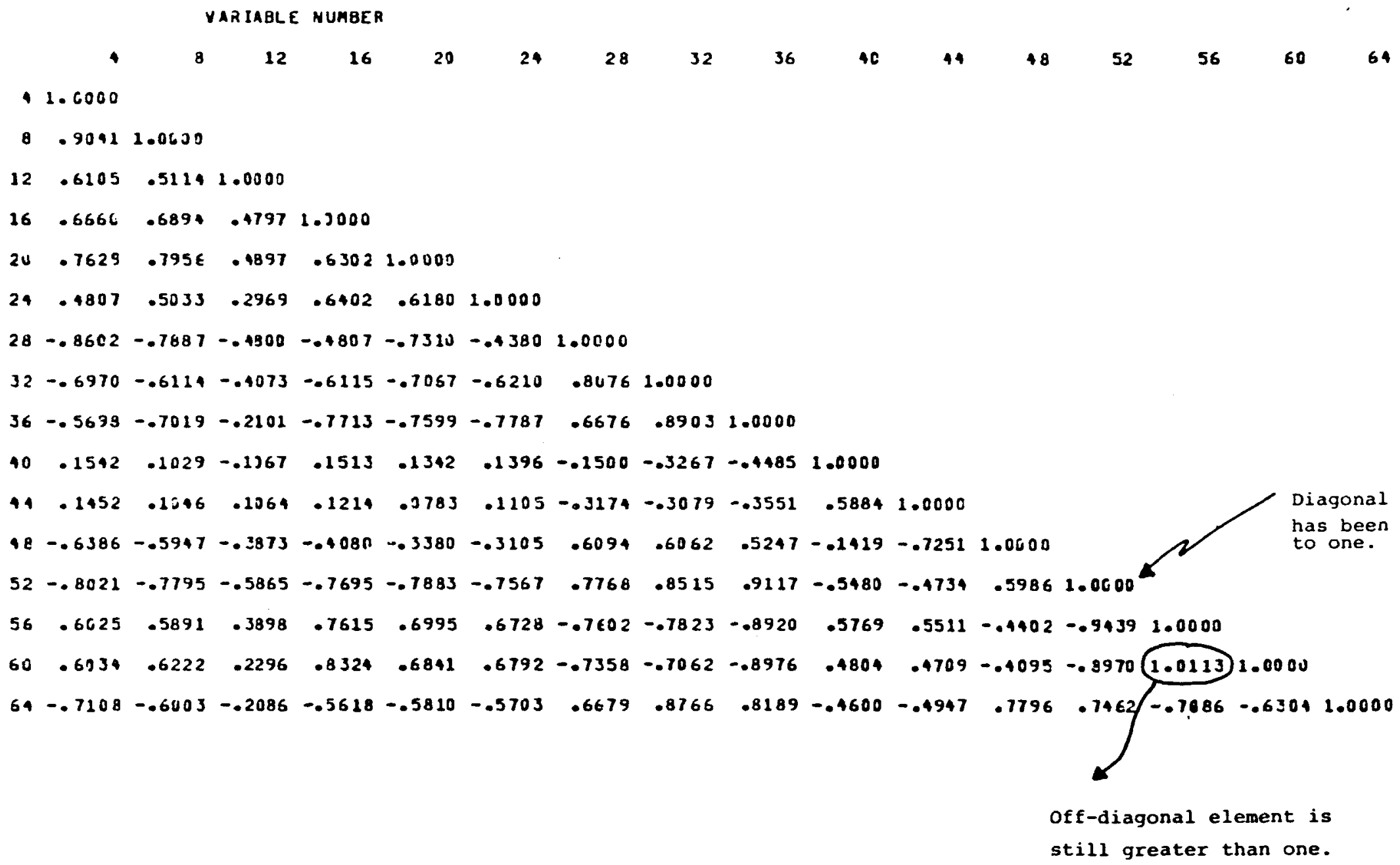


ITERAII ON MUMBER $=$ Beginning of the 12 th Iteration

INPLT - RANX CORRELATION STRUCTURE- FOR THE RANDOM VAR IABLES BEING TRANSF ORMED

VAR IABLE MURBER

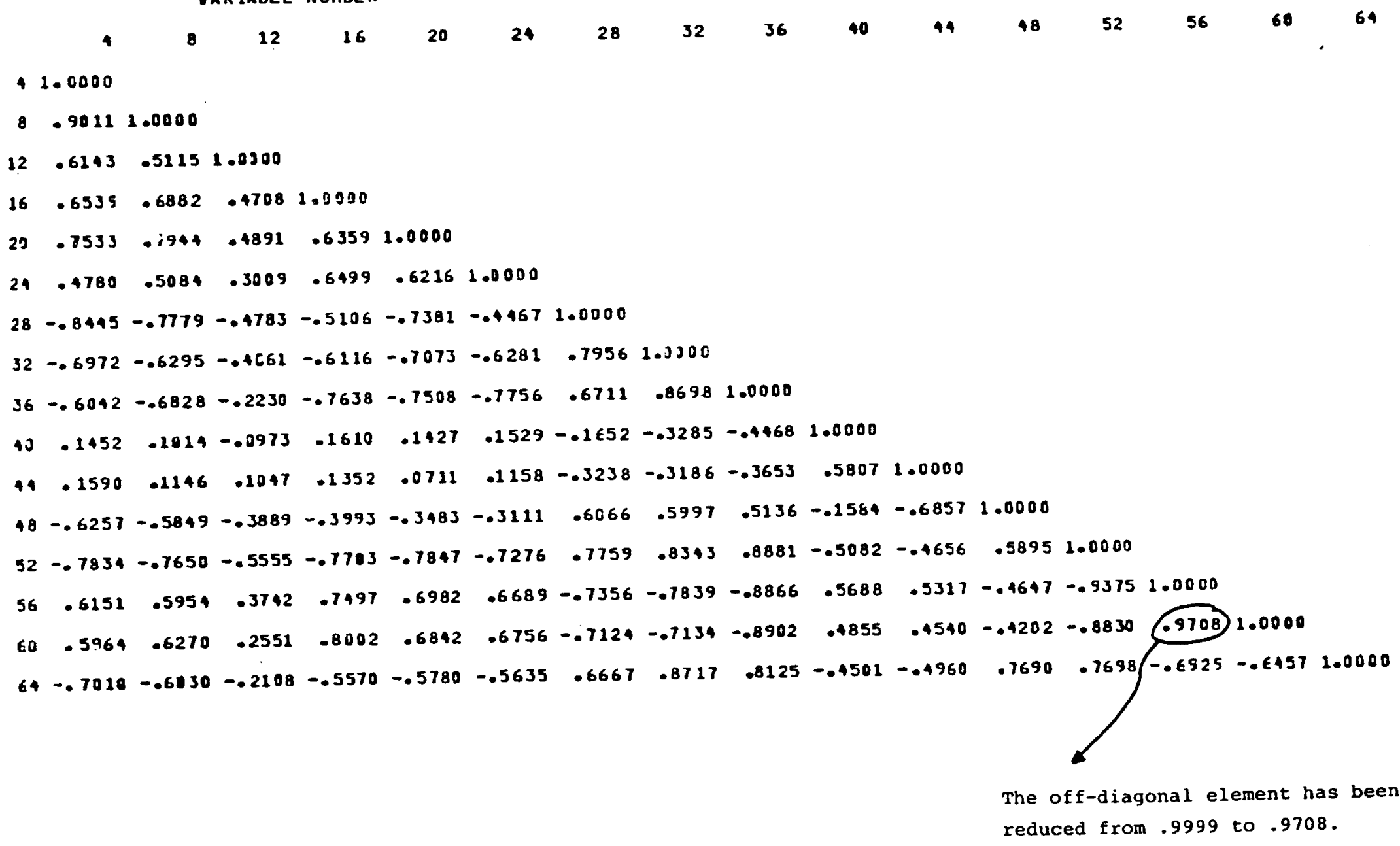

The off-diagonal element has been reduced from .9999 to .9708 . 
ANALIS

PERFORAANCE InOSX $=.125$

EIGENYAL UES =

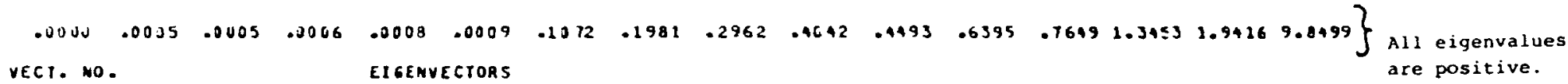

\begin{tabular}{|c|c|c|c|c|c|c|c|c|c|c|c|}
\hline $\begin{array}{c}1-5-983 E-c 2 \\
-.902\end{array}$ & $\begin{array}{l}-.124 \\
-7.06 \text { \&E }-02-\end{array}$ & $\begin{array}{c}-.213 \\
2-8.456 \varepsilon-02-\end{array}$ & $\begin{array}{l}-3.43 B E-02 \\
-2.391 E-02\end{array}$ & $-4.010 c-0=$ & -.178 & $4.849 E-02$ & $6.806 \varepsilon-0 z$ & .101 & -.231 & $-7.517 E-02-$ & $-1 .+00[-03$ \\
\hline $\begin{array}{l}2.315 E-02 \\
3.506 E-02\end{array}$ & $\begin{array}{l}.127 \\
.826\end{array}$ & $\begin{array}{l}-1.563 \varepsilon-02 \\
-.181\end{array}$ & $\begin{array}{l}-.248 \\
-.110\end{array}$ & $-1.462 E-C 2$ & $-9.049 E-02$ & .269 & .113 & $2.96 E E-02$ & -.153 & -.220 & -.234 \\
\hline $\begin{array}{l}324 \\
-5.731 E-03^{-5}\end{array}$ & $\begin{array}{l}-9.783 E-122 \\
-.183\end{array}$ & $\begin{array}{l}.268 \\
.544\end{array}$ & $\begin{array}{l}-.397 \\
-.239\end{array}$ & -.125 & -.166 & .192 & .264 & -.416 & -.127 & -.143 & $2.072 E-02$ \\
\hline $\begin{array}{l}-330 \\
3.573 E-63\end{array}$ & $\begin{array}{l}-.310^{\circ} \\
5.50 \cdot E-02\end{array}$ & $\begin{array}{l}-3.995 E-02 \\
2=-465\end{array}$ & $\begin{array}{l}.100 \\
.231\end{array}$ & $-3.926 \varepsilon-12$ & $-4.225 E-82$ & -.180 & .244 & -.637 & $2.035 E-0:$ & $7.170 E-02$ & $8.613 E-03$ \\
\hline $5: 904$ & $\begin{array}{l}.202 \\
-2.323 E-03-\end{array}$ & $\begin{array}{l}-8.860 E-02- \\
J-2.180 E-02\end{array}$ & $\begin{array}{l}-9.3 J 3 E-02 \\
7.605 E-02\end{array}$ & -.122 & $-2.201 E-02$ & .366 & -.352 & $-2.076 E-02$ & -.270 & .463 & .460 \\
\hline$=0.320$ & $\begin{array}{l}-.248 \\
-2.147 E-02\end{array}$ & $\begin{array}{r}-.150 \\
.239\end{array}$ & $\begin{array}{l}-9.592 E-02 \\
-5338\end{array}$ & -131 & $3.481 E-03$ & .284 & -.308 & $6.796[-02$ & .116 & -.196 & $-.45 E$ \\
\hline $\begin{array}{r}7: 394 \\
.111\end{array}$ & $\begin{array}{l}-.515 \\
7.794 E-12\end{array}$ & $\begin{array}{r}-9.112 x-02 \\
8.728 E-02\end{array}$ & -.151 & .370 & $4.889 \varepsilon-02$ & $3.915 E-02$ & .267 & .378 & $-8.865 E-02$ & .105 & $9.747 E-02$ \\
\hline $\begin{array}{l}8.360 \\
-6.11 \leq \varepsilon-02^{-}\end{array}$ & $\begin{array}{l}-2.247 E-02- \\
5.873 E-02\end{array}$ & $\begin{array}{l}2-6.588[-02 \\
2.117\end{array}$ & $\begin{array}{l}-.116 \\
3.731 E-02\end{array}$ & -.660 & .104 & -.351 & $9.2 * \varepsilon-02$ & .249 & $5.259 E-02$ & $2-. .153$ & $7.396 E-0 z$ \\
\hline $\begin{array}{l}9.300 E-02 \\
-.105\end{array}$ & $\begin{array}{r}.325 \\
\therefore .125\end{array}$ & $\begin{array}{l}-.132 \\
-9.931 E-02\end{array}$ & $\begin{array}{r}-.009 \\
.156\end{array}$ & .349 & .496 & .115 & .455 & $2.634 E-62$ & $5.692 E-02$ & .217 & $-9.317 E-02$ \\
\hline $\begin{array}{l}10-250 \\
B .271 E-02\end{array}$ & $\begin{array}{r}-.195 \\
.236\end{array}$ & $\begin{array}{r}-.110 \\
.288\end{array}$ & $\begin{array}{r}-.271 \\
.384\end{array}$ & .150 & $-3,+16 E-3$ & -.512 & $-6.332 E-8:-$ & {$[-8.090[-03$} & -.465 & .259 & $5.85 \Xi E-02$ \\
\hline $\begin{array}{ll}11 & -161 \\
--108 & 108\end{array}$ & $\begin{array}{l}-. .374 \\
-1.890 E-122\end{array}$ & $2-.397$ & $\begin{array}{l}-.461 \\
-.155\end{array}$ & .128 & .279 & $-1.943 E-22$ & $2-.441$ & $5.6: 9[-0]$ & .147 & -.183 & .163 \\
\hline $12-0.236$ & $1.0935-02$ & $2 \begin{array}{l}-.233 \\
3.2905-03\end{array}$ & $\begin{array}{l}-.316 \\
3.288 \varepsilon-02\end{array}$ & .259 & -.488 & -.335 & $-2.648 E-12$ & $87.8696-02$ & .417 & -.206 & .317 \\
\hline $\begin{array}{c}13-1.5035-02- \\
-.194\end{array}$ & $\begin{array}{l}-6.4565-32 \\
.226\end{array}$ & $\begin{array}{l}.627 \\
.132\end{array}$ & $\begin{array}{l}.164 \\
.422\end{array}$ & $-1.757 E-02$ & $2-.10$ & $7.128 E-02$ & .260 & .205 & .256 & .221 & .205 \\
\hline $\begin{array}{l}14-.218 \\
-4.9625-02\end{array}$ & $\begin{array}{r}-.108 \\
.177\end{array}$ & $\begin{array}{r}-.279 \\
.252\end{array}$ & $\begin{array}{l}.226 \\
.149\end{array}$ & .136 & .351 & .193 & $1.362 E-02$ & $=-.235$ & .128 &.- .364 & .556 \\
\hline $\begin{aligned} 15 & -.251 \\
-4.144 E-U 2 & \end{aligned}$ & $\begin{array}{r}-.269 \\
.167\end{array}$ & $\begin{array}{r}-.349 \\
.133\end{array}$ & $\begin{array}{l}-.142 \\
-.166\end{array}$ & -.239 & $-7.282 E-02$ & .118 & $-1.469 E-\cup 2$ & $2-. .101$ & .534 & .525 & -.105 \\
\hline 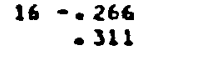 & $\begin{array}{l}-.261 \\
-.291\end{array}$ & $\begin{array}{l}-.160 \\
-.282\end{array}$ & $\begin{array}{r}-.252 \\
.268\end{array}$ & -.260 &.- .229 & .269 & .283 & .252 & -.227 & -.239 & .213 \\
\hline
\end{tabular}


ANALYSIS OF THE 2 BY 2 PRIME OIAGONAL SUBAAIRIX

PERFORAANCE INDEX $=.132$

EIGENYAL UES =

.09891 .9011

VECT. MO.

EI GENVECTORS

$$
\begin{array}{rr}
1-.707 & .707 \\
2-.707 & -.707
\end{array}
$$

DETERTINANT $=.188$

Figure 7 
AMALYSIS OF THE 3 BY 3 PIIME DIAGONAL SUBMATRIX PERFORMA NCE INDEX $=.255$

EIGENYALUES =

$.0895 \quad .5446 \quad 2.3659$

VECT. NO.

EI GENVECTORS

$\begin{array}{lll}1.0 .740 & .660 & .128 \\ 2 . .256 & -.053 & .854 \\ 3 . .622 & .599 & .504 \\ \text { ERKINANT }= & .115 & \end{array}$


V. Deck Setup for POSDEF

Following we give an example of how to set up the cards for use in running POSDEF. The first 10 cards are the usual job card, account card, and other control cards. itiis is followed by the Fortran program which is followed by the data (a listing of the progran is given in Appendix A).

\title{
DECK SETUP FOR POSDEF
}

\author{
YPO,T5, STSSZ. \\ USER, NAMEXXX, PASWORD. \\ CHARGE, 0188000 . \\ $F T N, R=2$. \\ PFGET, SUBZ, OLDLIB , AU=ELFROST. \\ PFGET, IMSL , FXIMSL , AU =MATHLIB. \\ LIBRARY, SUBZ, IMSL. \\ LUSET, PRESET $=$ NGINDEF. \\ $L G 0, P L=10000$. \\ EXIT. \\ EXIT.
}

(END OF RECORD -- MULTI-PUNCH 789 IN COL 1)

PROGRAM POSDEF GOES HERE

(END OF RECORD -- MULTI-PUNCH 789 IN COL 1) 
[DATA AND INPUT-PARAMETER CARDS GO HERE]

(END OF RECORD -- MULTI-PUNCH 789 IN COL 1)

(END OF INFORMATION -- MULTI-PUNCH 6789 IN COL 1)

This completes the information needed to run POSDEF. An example of the data that was used in the example in section IV is given in Figure 9. 
Figure 9

Input-Parameter Cards

Card

No.

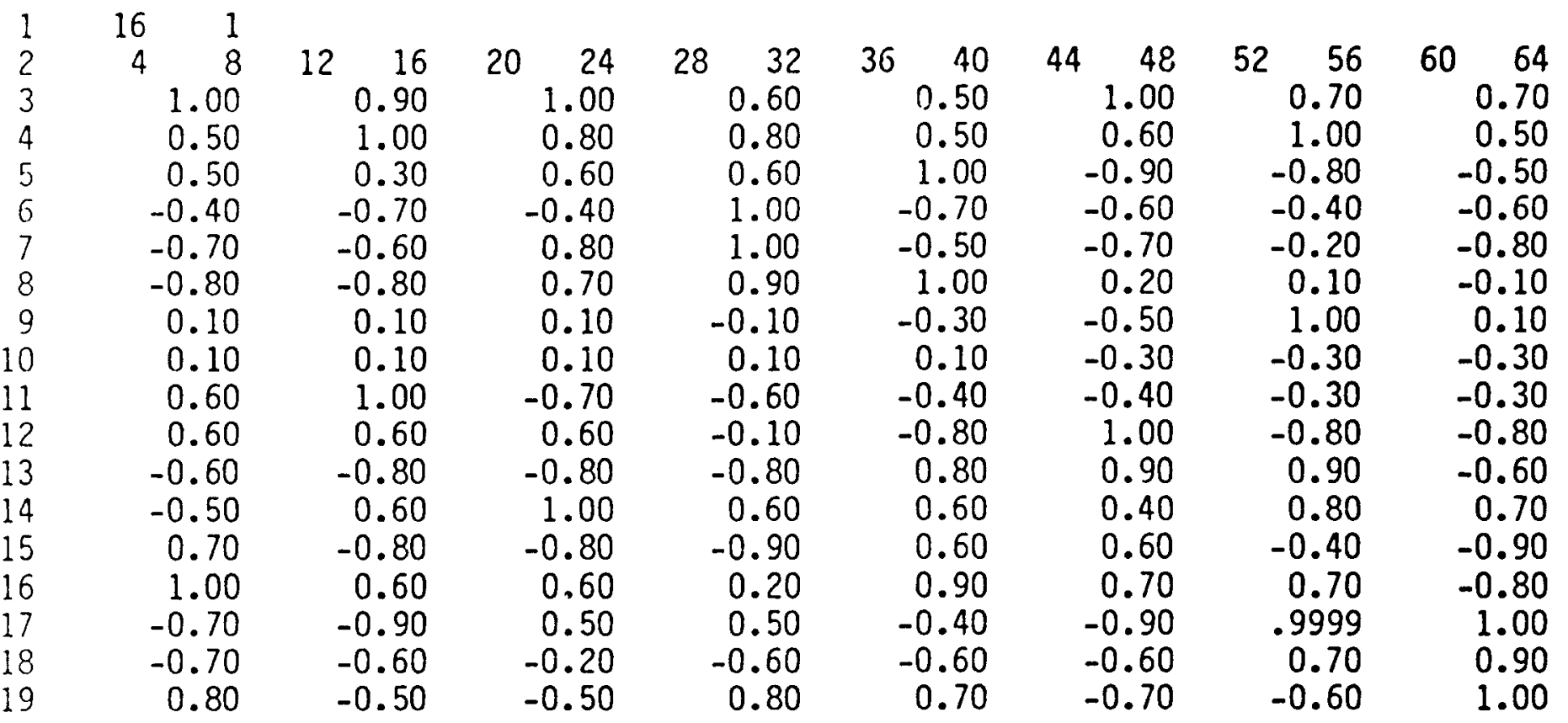


VI. Discussion

The user should recall that the input "approximate correlation matrix" can be ill-conditioned. These types of matrices frequently occur when a modeler places positive ones on the diagonal elements and then assigns correlations to the pairwise configurations of variables. While it is impossible to construct a negative definite matrix in this way, this matrix will have only a few negative eigenvalues. These will be small in absolute value (very near zero), provided this matrix is not 111 -conditioned (i.e. one or more negative eigenvalues that are relatively large in absolute value such as $-1,-2$ or -3 ).

Recall that we have no guarantee that this algorithm will converge in such ill-conditioned cases, but we have never failed to see it converge. However, the final correlation matrix may be quite different from the original "approximate correlation matrix." We illustrate some of these principles with the following example.

Figure 10 gives the input "approximate rank correlation matrix" for 19 variables, labeled as indicated. As can be seen, the modeler indicates that variable numbers 1 through 11 are independent of each other. Also variable numbers 22 and 23 are each correlated negatively with each of variables 1-11. The indicated value of this correlation coefficient is -0.7. Finally the modeler indicates that variables 22 and 23 are independent. 
A careful examination of these relationships will show that such an arrangement is essentially impossible. Consider the three hypothetical variables $x_{1}, x_{2}$ and $x_{3}$. Suppose $\left(x_{1}, x_{2}\right)=-0.7$ and furthermore suppose $\left(x_{1}, x_{3}\right)=-.8$. Then it is obvious that $\left(x_{2}, x_{3}\right)>0$; that is variables $x_{2}$ and $x_{3}$ will have a strong positive correlation.

This matrix is ill-conditioned. There is only one negative eigenvalue hut its value is -2.3571 . However, the algorithm did converge after eight iterations. But it is not surprising that the values of the original matrix have been altered quite a bit. For example, the correlation value -0.7 becomes -0.4033 ; the zero correlation between variables 22 and 23 becomes 0.6451 ; and the zero correlations assigned to variables 1-11 all become 0.1214 (see Figure 11 ). This may or may not be acceptable to the modeler, but does indicate that care should be taken in the construction of the original "approximate correlation matrix." This simply illustrates that a collection of pairvise correlation coefficients does not make a correlation matrix. A multivariate distribution is much more complicated than this. 
INPUT -RANK CCRRELATION STRUCIURE FOR THE RANCOA VARIABLES GEING TRAMSFORMED

$$
\text { VARIABLE NUMUER }
$$

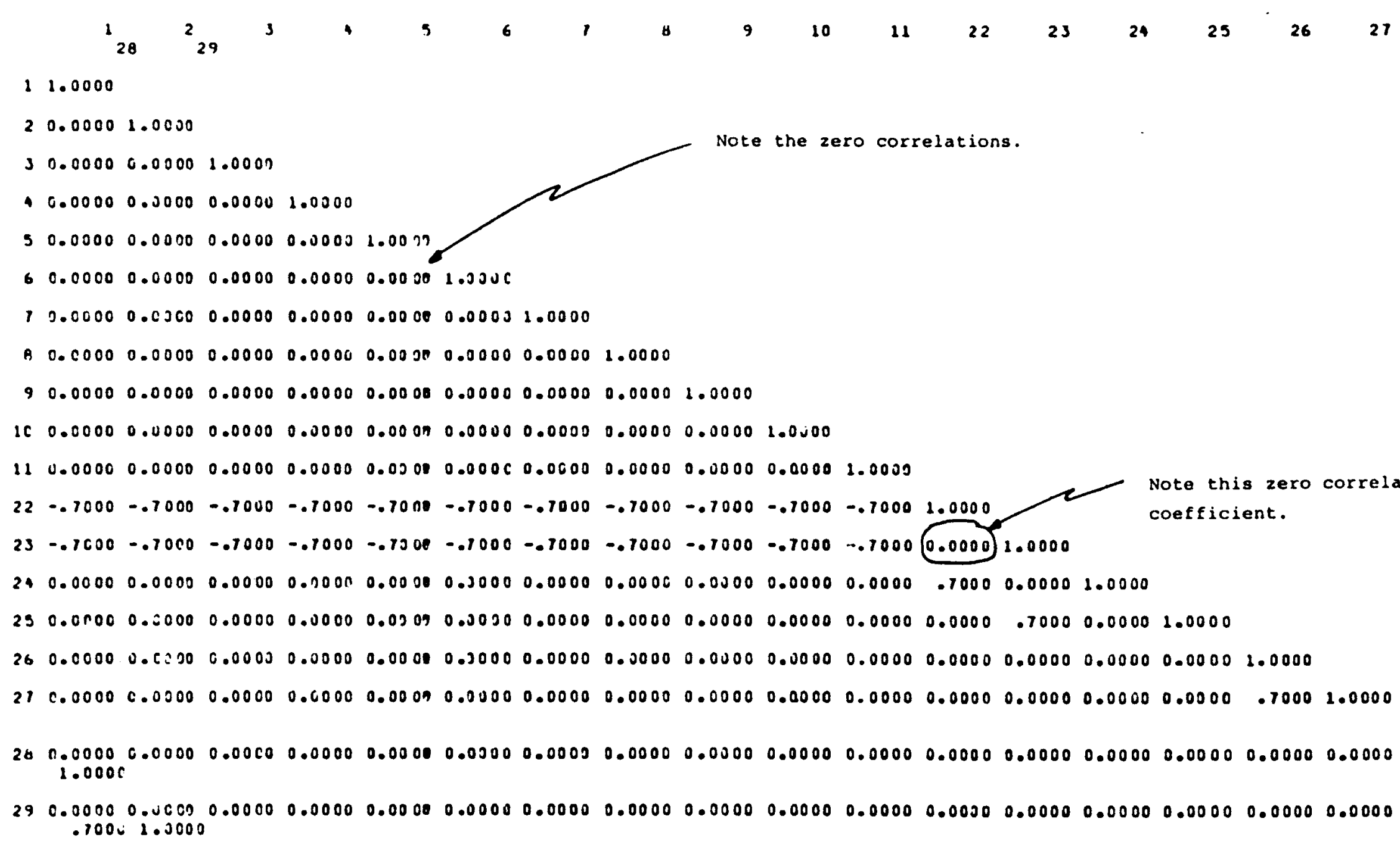


INPUT FRANK CORRELAIION STRUCTURE - For THE RANCOM VARIABLES BEING IRANSFORMED

VARIARLE MUMBER

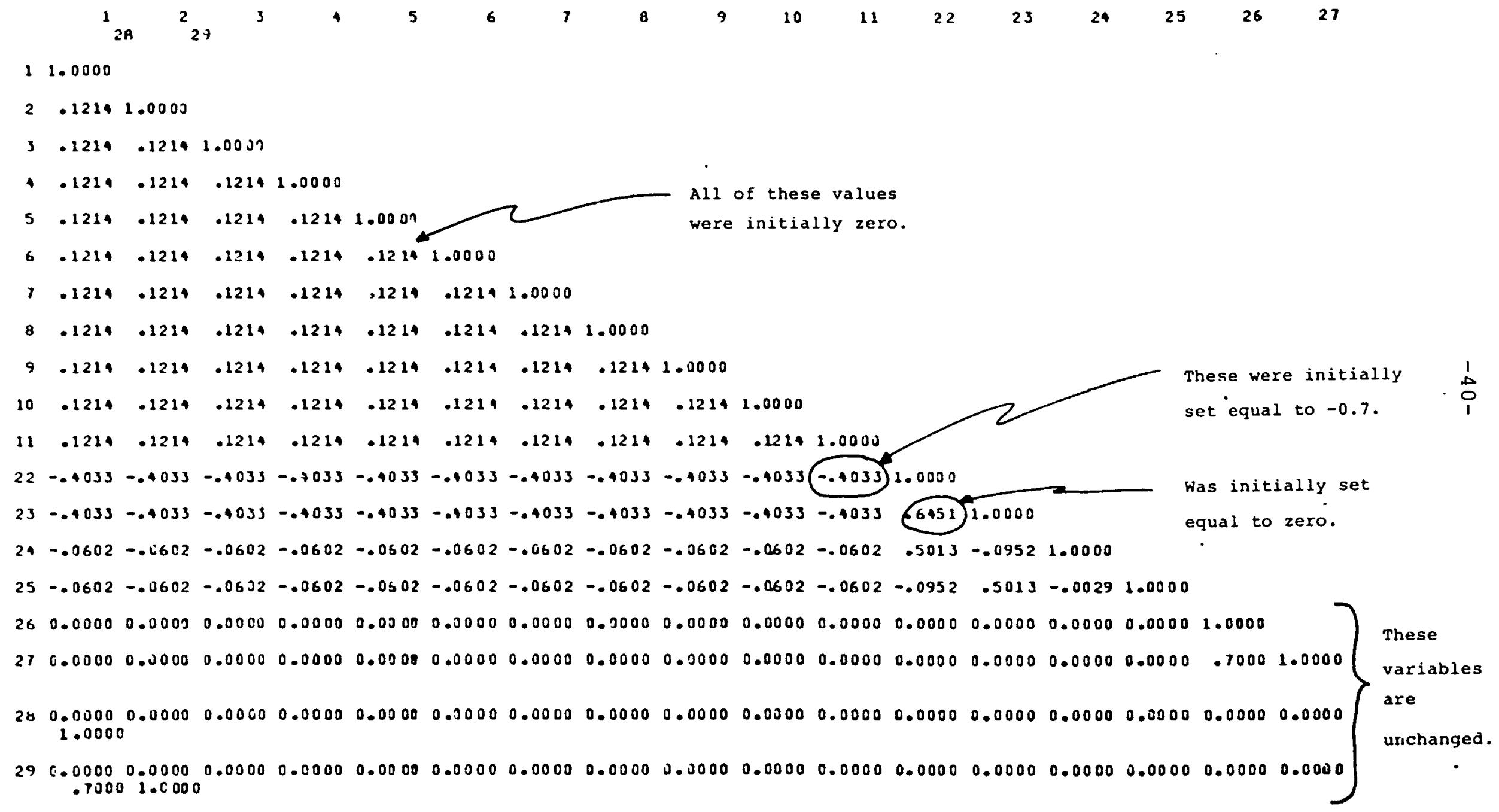


Graybill, F. A. (1961). An Introduction to Linear Statistical Models, Volume 1, New York City, McGraw-Hill Book Company, Inc.

IMSL (1980). The IMSL, Volume 1, Ed. 8, Houston, Texas, International Mathematical and Statistical Libraries, Inc.

Iman, R. L. and Davenport, J. M. (1980). Rank Correlation Plots for Use with Correlated Input Variables in Simulation Studies. Tech Report SAND80-1903, Sandia Laboratories, Albuquerque, NM. 
$-42-$

THIS PAGE LEFT BLANK INTENTIONALLY 
$-43-$

APPENDIX A. Listing of the Fortran Source Deck for the POSDEF Program. 
$-44-$

T'HIS PAGE LEFT BLANK INTENTIONALLY 
PROGRAM POSDEF (INPUT, OUTPUT, PUNCH, TAPE5)

DIMENSION CORX (210), NK(20), D (20), Z(20,20), WK(210), CORZ(210)

$C$
$C$
$C$
$C$
$C$
$C$
$C$
$C$

C NN = THE DIMENSIONS OF Z, I.E. Z(NN,NN).

DATA NN/20/

C EIG = THE VALUE THAT THE NEGATIVE EIGENVALUES ARE SET EQUAL TO.

DATA EIG/0.001/

C

$M=$ MAXIMUM NUMBER OF ITERATIONS ALLOWED.

$M=20$

$M I=M+1$

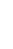

ITEST $=1$

READ 110, NP, IDIAG

READ 120, (NK(I), I =1,NP)

$N K X=N P *(N P+1) / 2$

READ 130, (CORX(I), I=1,NKX)

10 PRINT 140

IF (ITEST.GT.M) GO TO 20

GO TO 30

20 IF (IDIAG.EQ.0) GO TO 100

PRINT 150

GO TO 40

30 PRINT 160, ITEST

40 PRINT 170

PRINT 180

PRINT 190, (NK(I), I =1,NP)

II $=1$

IK $=1$

DO $50 \quad I=1, N P$

PRINT 200, NK(I), (CORX (J),J $=I I, I K)$

$I I=I K+I$

$I K=I K+I+1$

50 CONTINUE

$\mathrm{IL}=\mathrm{NP}$

IF (ITEST.EQ.MI) IL=2

DO $80 \mathrm{~K}=\mathrm{IL}$, NP

CALL EIGRS (CORX, K, 2,D,Z, NN,WK, IER)

PRINT 210, K,K

PRINT 220, WK(1)

PRINT 230, (D(I), I =1,K)

PRINT 240

DO $60 \mathrm{~J}=1, \mathrm{~K}$

60 PRINT 250, J, $(Z(I, J), I=1, K)$

$D E T=1.0$

DO $70 \mathrm{~J}=1, \mathrm{~K}$

$70 D E T=D E T * D(J)$ 


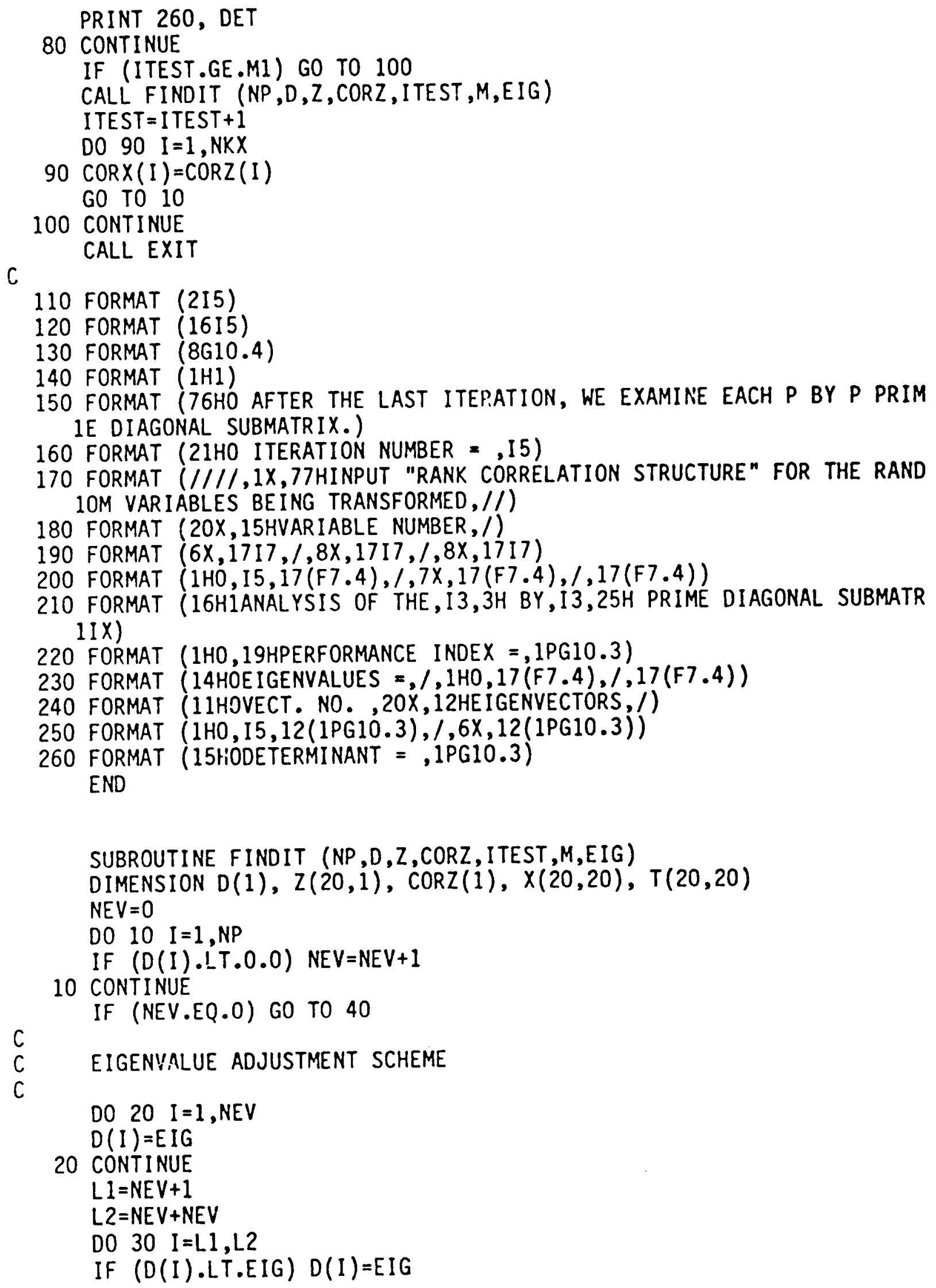




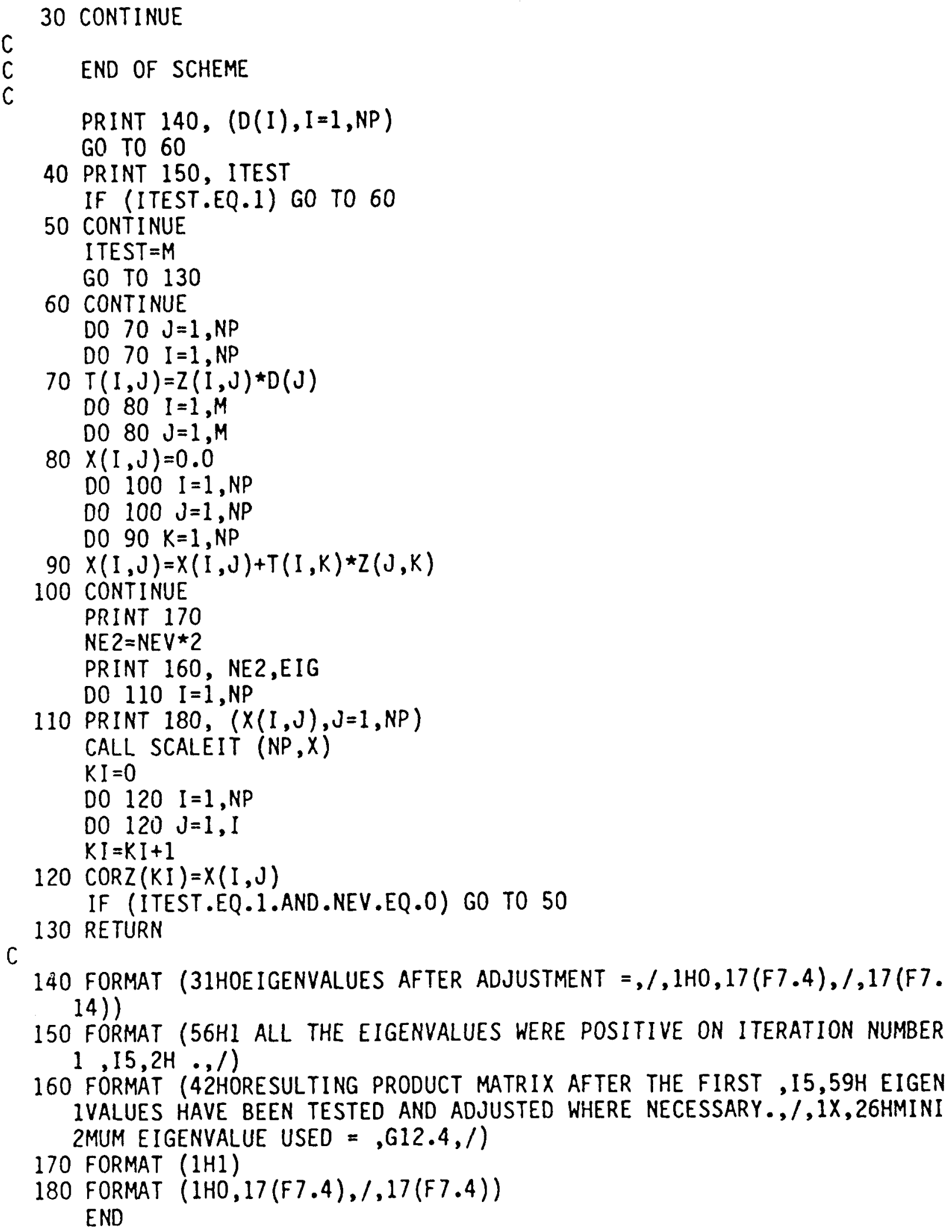




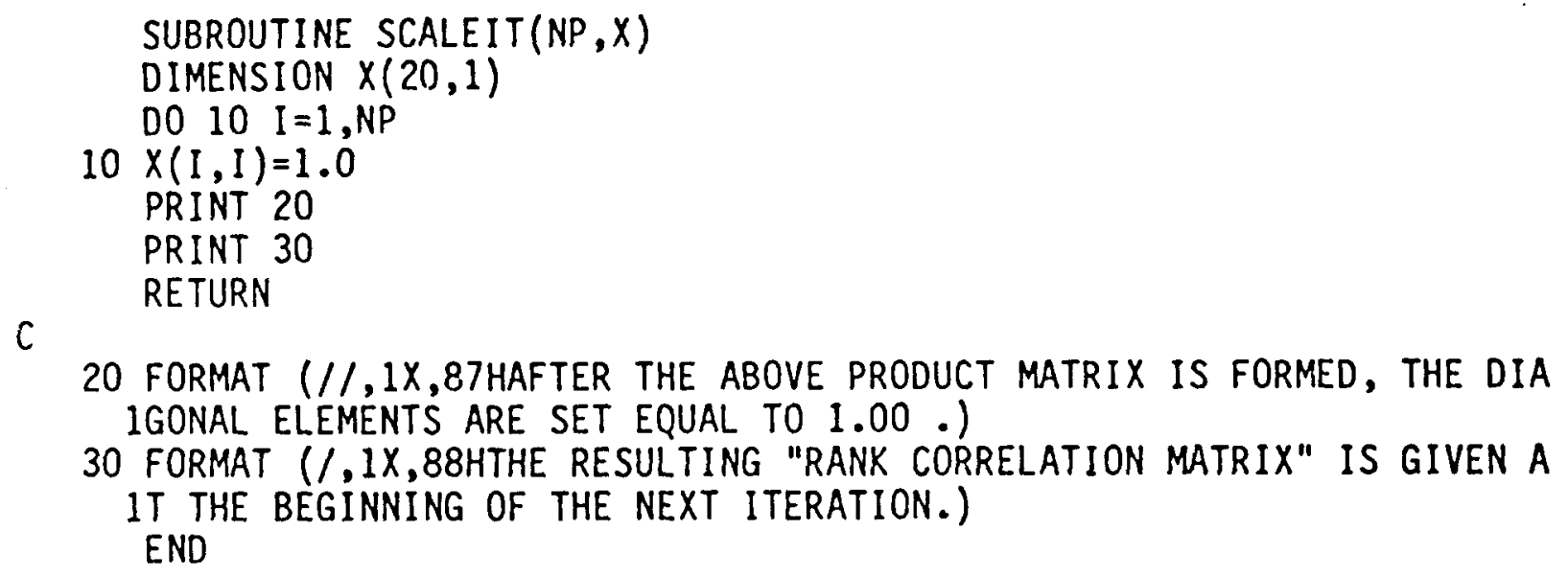

20 FORMAT $(/ /, 1 X, 87$ HAFTER THE ABOVE PRODUCT MATRIX IS FORMED, THE DIA IGONAL ELEMENTS ARE SET EQUAL TO 1.00 .)

30 FORMAT (/,1X,88HTHE RESULTING "RANK CORRELATION MATRIX" IS GIVEN A 1T THE BEGINNING OF THE NEXT ITERATION.) END 
Distribution:

Probabilistic Analysis Staff (25)

Office of Nuclear Regulatory Research

U.S. Nuclear Regulatory Commission

Washington, D. C. 20555

Attn: M. Cullingford

Clycle Jupiter

NRC/?ES

U.S. Nuclear Regulatory Commission

Washington, D. C. 20555

Daniel Egan

Office of Radiation Programs (ANR-460)

IJ.S. Environmental Protection Agency

Washington, D. C. 20460

Los Alamos Scientific Lab. (4)

Group S1, MS 606

Los Alamos, NM 87545

Attn: M. D. Mckay

R. A. Waller

R. J. Beckman

M. E. Johnson

U.S. Geologic Survey (2)

U.S. Dept. of Interior

P.0. Box 25046

Denver Federal Center

Denver, CO 80225

Attn: R. Waddel1, MS 416

Nuclear Hydrology Projcct, WRD

W. S. Twenhofel, Chief

Special Projects Branch

W. J. Conover

College of Business Administration

P. 0. Box 4320

Lubbock, TX 79409

J. M. Davenport (10)

Department of Mathematics

P.0. Box 4319

Lubbock, TX 79409 


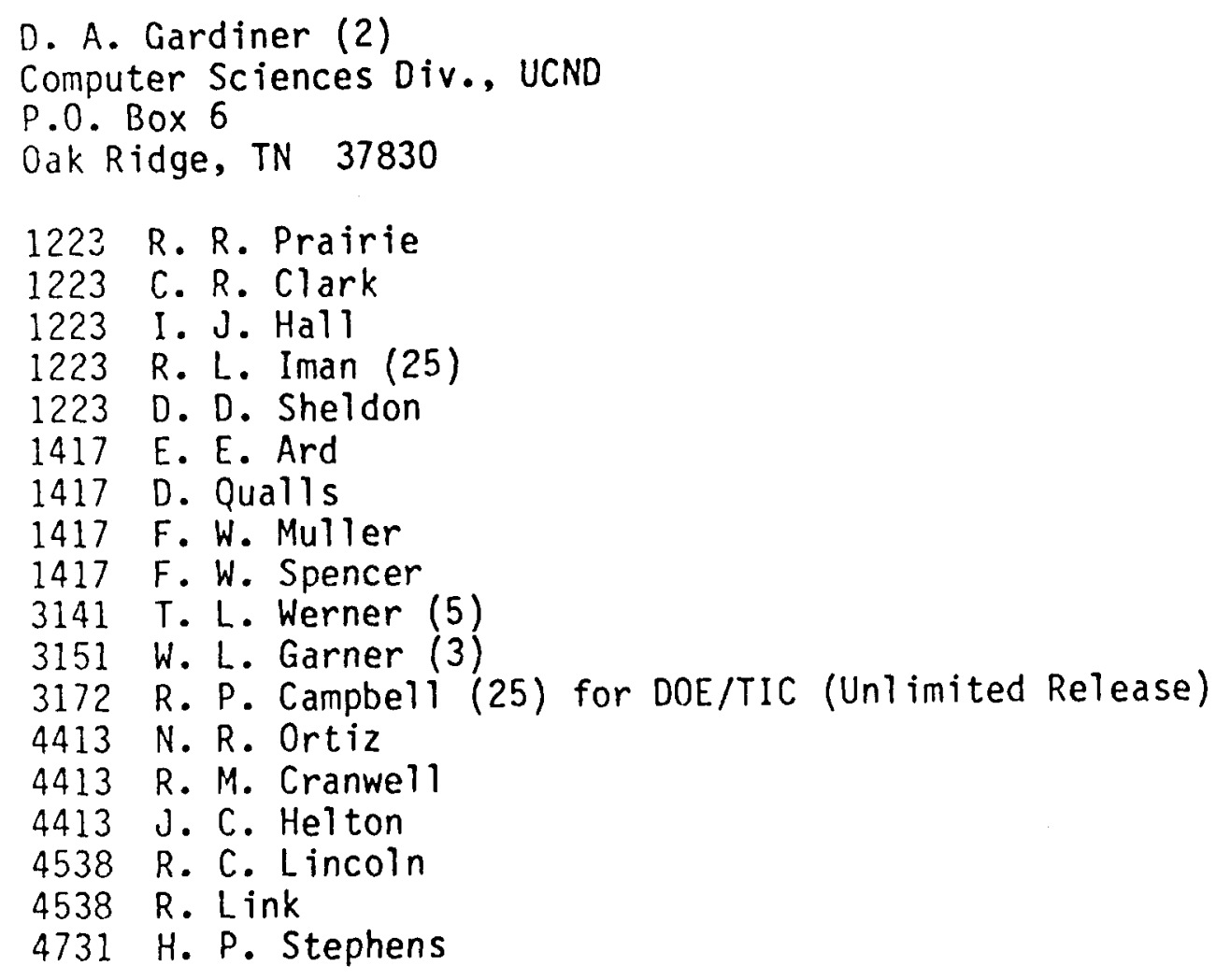

